\title{
Lighting control method based on the natural illumination model and the personnel movement model
}

\author{
Qiansheng Fang ${ }^{1}$, and Chang $\mathrm{Tu}^{1}$, Chenlei Xie ${ }^{1, *}$ \\ ${ }^{1}$ Anhui Province Key Laboratory of Intelligent Building and Building Energy Saving, Anhui JianZhu University, Hefei, China
}

\begin{abstract}
Since the light control module and communication module connected by the CAN bus, the traditional and centralized lighting control system has a high cost of maintenance. Meanwhile, the connecting way relied on the CAN bus is hard to achieve energy saving. It's entirely possible to improve the efficiency by using the design of distributed non-central platform, we proposed a new lighting control simulation system based on the natural illumination model and the personnel movement model. Considering that all influencing factors of the real light environment, this paper calculated the illumination of light source on the working surface and the natural illumination in the room by the method of azimuth coefficient and boundary integral. Furthermore, in order to decrease energy consumption, we introduced the Markov transfer matrix to describe the position changes of staff to control the light intensity of the office. Though the simulation results, the proposed lighting control system in this paper is demonstrated that can save more lighting energy consumption in comparison with traditional and centralized lighting control system.
\end{abstract}

\section{Introduction}

Generally, Centralized lighting control system is a network control method which consists of control bus, control switch and the output circuit. Since the complex connections, Centralized lighting control system is hard to meet the demand of intelligent control and energy saving. ${ }^{[1]}$

It can be seen that the centralization of the system has hindered the development of intelligent control in buildings, ${ }^{[2]}$ therefore the building non-central platform is proposed by Qi shen in 2015 to efficiently share the underlying information to perform the self-identification, self-organization and self-coordination. As it is illustrated, the building non-central platform improves the efficiency and the expandability of the centralization system. ${ }^{[2]}$

Since the randomness and uncertainty of the electrical behaviour of equipment in buildings, the proportion of lighting energy consumption to the total energy consumption of office building is as high as $20 \%$ $40 \%$ by a large number of on-site investigations. And it is common that the electrical equipment such as lighting fixtures is still running when there is no one in the room. Therefore, lighting energy saving is one of the key links in office building energy conservation. ${ }^{[3-4]}$ However, the uncomplicated controlling methods and few equipments make the Energy saving is easier to be implemented and promoted than other systems in buildings, the measure to save energy is simple as using the LED energy-lamps to reduce power consumption. Energy saving measures are easier to implement and promote than other systems in buildings, such as air conditioning systems. Whereas above, we suggested adopting the automatic control systems to reduce unnecessary lamp opening time and efficiently achieve energy-saving effects. ${ }^{[5-6]}$

Lighting control is integral in the lighting system which is the main component of the lighting design. The switch of the circuit was the main content of the lighting control in the past. The scene was controlled only in the lighting of the stage and the multi-function banquet hall. With the development of the architectural science, intelligent lighting control has become an indispensable part of lighting design. For improving the performance of the intelligent lighting control and saving more energy than the tradition lighting control, in this paper, we proposed a new lighting control simulation system. The lighting control simulation system considering the weather (such as outdoor solar radiation level, solar elevation angle change), architectural features (such as office lighting, window area, orientation), personnel characteristics (such as the distribution of personnel) and so on, we calculated the illumination value attenuation mathematical model after attenuating the natural light indoors, then through the influence of the event on the personnel's position to use the natural light reasonably, so that the efficiency of the lamps and electrical energy has been improved. Effectively extend the lamp's life and reduce the energy consumption while ensuring sufficient quantity and quality of lighting.

\section{Establishment of an office natural lighting field model}


Since the complex light condition is difficult to be analyzed, in this paper, we designed an illumination field variation models to simulate the daily true light environment. The model is used to determine whether artificial light is needed at a certain time, and then turn on the light only when the illumination is insufficient. Furthermore, as the natural source, natural light is sustainable, energy-saving and environmentally friendly. Using natural light indoors directly can save unnecessary energy loss during photoelectric conversion and avoid the damage of waste to the environment..

The natural light shines from the window can be divided into two types: direct sunlight and diffuse sunlight. The direct sunlight refers to the light that the sun emits through the atmosphere and reaches the ground, and the diffuse sunlight refers to the light that the sun emits through multiple reflections, such as the vapour in the atmosphere, the thick cloud layer, etc. On the other hand, direct light can also form diffuse light through the diffuse reflection of objects such as trees and buildings. In cloudy days, most of the light through the window is diffusely reflected. Only few direct lights can penetrate the office without any blocks and reflections. Therefore, in the illumination value attenuation mathematical model, we can use the plane of the window as a Lambertian radiator with an intensity of $E$ to facilitate the quantitative calculation of the illumination at each point in the room. The room diagram in this model is shown in Fig 1:

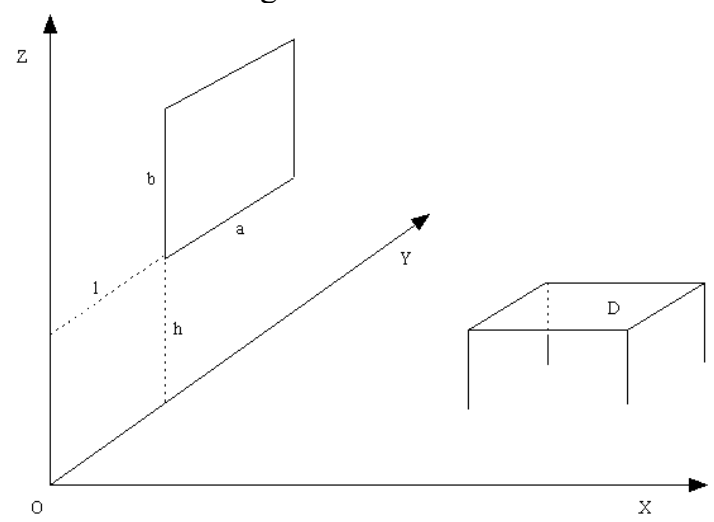

Fig. 1.The sketch map of indoor natural light.

The corner of the room on the side of the window is taken as the coordinate origin, and the wall in the horizontal direction is the XY coordinate system. Natural light shines into the room through windows with length $a$ and width $b$, respectively. The lower edge of the window is $h$ from the ground and the distance along the corner of the room is $l$. The illumination produced by such a uniformly illuminated surface light source at point $\mathrm{D}$ of the working surface is $E_{D}$.

The above model is obtained in the illumination calculation problem of a rectangular surface light source, as shown in Fig 2:

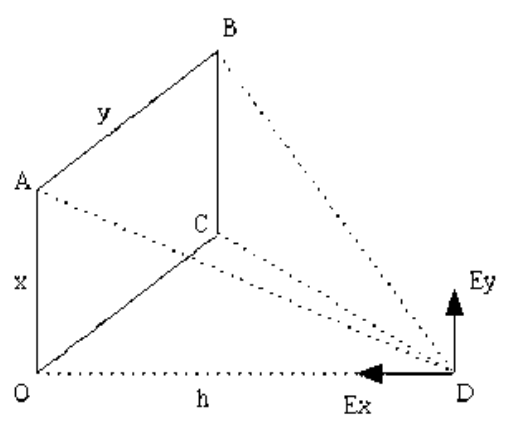

Fig. 2.The computing method of rectangular surface light.

In the Fig 2, the luminous intensity of the surface light source is $L$, the illuminated point is at the apex of the light source, and the distance is $h$, the angle formed by the element and illuminated face is $\delta$, the horizontal illumination of point $\mathrm{D}$ is $E y$, and the vertical illumination is $E x$, then the illumination at point $\mathrm{D}$ is superimposed by the four sides of $\mathrm{OA}, \mathrm{AB}, \mathrm{BC}$ and $\mathrm{CO}$.

For the side OA:

$$
\begin{gathered}
\beta_{1}=\arctan (x / h), \quad \delta_{1}=\pi / 2 \\
\operatorname{So:} \cos \delta_{1}=0
\end{gathered}
$$

For the side AB:

$$
\begin{gathered}
\beta_{2}=\arctan \left(y / \sqrt{\mathrm{x}^{2}+h^{2}}\right), \delta_{2}=\arctan (h / x) \\
\text { So:cos } \delta_{2}=x / \sqrt{x^{2}+h^{2}}
\end{gathered}
$$

For the side $\mathrm{BC}$ :

$$
\begin{gathered}
\beta_{3}=\arctan \left(x / \sqrt{\mathrm{x}^{2}+h^{2}}\right), \quad \delta_{3}=\arctan (h / y) \\
\text { So:cos } \delta_{3}=y / \sqrt{y^{2}+h^{2}}
\end{gathered}
$$

For the side $\mathrm{CO}$ :

$$
\begin{gathered}
\beta_{4}=\arctan (y / h), \quad \delta_{2}=\pi / 2 \\
\operatorname{So}: \cos \delta_{4}=0
\end{gathered}
$$

So the vertical illumination of point $\mathrm{D}$ can be obtained:

$$
\text { Ey }=\frac{\mathrm{L}}{2}\left(\frac{x^{2}}{\sqrt{x^{2}+h^{2}}} \arctan \frac{x}{h}-\frac{h}{\sqrt{x^{2}+h^{2}}} \arctan \frac{x}{\sqrt{y^{2}+h^{2}}}\right)
$$

According to the horizontal illumination of point D and luminous intensity of the form, the illumination value at each point of the office can be obtained.

\section{Establishment of lighting illumination field model}

After obtaining the natural illumination model, it is necessary to simulate the illumination produced by the office lighting used on the work surface. When the natural light conditions are not up to standard, the illumination of the part is superimposed after turning on a light to obtain a comprehensive illumination. Since the lighting fixtures are fluorescent tubes that widely used in offices, classrooms and hospitals, so each one can be approximated as a linear, uniform illuminating light source.

Fig 3 shows the illumination calculation at point $\mathrm{P}$ when the line source $A B$ is on a horizontal plane: The point $\mathrm{P}$ is aligned with one end of the line source, the angle formed by the normal to the horizontal plane and the incident light plane APB is $\beta$. 


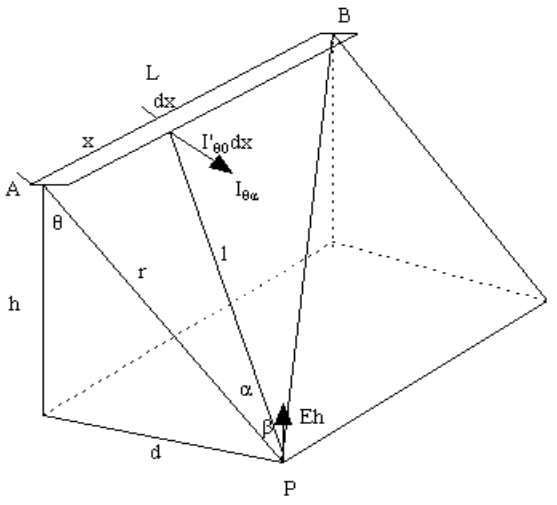

Fig.3.Illumination of linear light source at point $P$.

Assuming that the lighting unit $d x$ on the line source of length $L$, the unit length light intensity of the line light source on the plane APB perpendicular to the direction of the lamp axis $\mathrm{AB}$ is I' ${ }_{\theta 0}=\mathrm{I}_{\theta 0} / \mathrm{L}$, the light intensity of the $d x$ points to the point $\mathrm{P}$ :

$$
\mathrm{dI}_{\theta \alpha}=\frac{\mathrm{I}_{\theta 0}}{\mathrm{~L}} \mathrm{dx} \cos ^{n} \alpha=I_{\theta \alpha}^{\prime} \mathrm{dx} \cos ^{n} \alpha
$$

The normal illumination of the line element $\mathrm{dx}$ at point $\mathrm{P}$ :

$$
d E_{n}=\frac{\mathrm{dI}_{\theta \alpha}}{l^{2}} \cos \alpha=\frac{\mathrm{I}_{\theta 0 \mathrm{dx}} \cos ^{n} \alpha \cos \alpha}{L * l^{2}}
$$

The normal illumination En generated by the entire line source at point $\mathrm{P}$ :

$$
E_{n}=\int_{0}^{\alpha_{1}} \frac{\mathrm{I}_{\theta 0} \mathrm{dx} \cos ^{n} \alpha \cos \alpha}{L * l^{2}}
$$

Fig 2-1 shows that:

$$
x=r \tan \alpha
$$

Then:

$$
\begin{gathered}
d x=r \sec ^{2} \alpha d \alpha \\
\frac{r}{l}=\cos \alpha \\
l=\frac{r}{\cos \alpha} \\
\cos \beta=\cos \theta=\frac{h}{r}
\end{gathered}
$$

Bringing the above relationship into (1), we can get:

$$
E_{n}=\frac{\mathrm{I}_{\theta 0}}{\mathrm{Lr}} \int_{0}^{\alpha_{1}} \cos ^{n} \alpha \cos \alpha d \alpha
$$

The horizontal illumination Eh of point $\mathrm{P}$ can be calculated:

$$
E_{h}=E_{n} \cos \beta=\frac{\mathrm{I}_{\theta 0}}{\mathrm{Lr}} * \frac{\mathrm{h}}{\mathrm{r}} F_{x}=\frac{\mathrm{I}_{\theta 0}}{\mathrm{Lr}^{2}} F_{x}=\frac{I_{\theta 0}^{\prime}}{h} \cos ^{2} \theta F_{x}
$$

$I_{\theta 0}$ is the light intensity of the line light source on the plane APB perpendicular to the direction of the lamp axis $\mathrm{AB}$, and $I_{\theta 0}^{\prime}$ is the unit length light intensity, the unit is cd; $L$ is the length of the linear luminaire, the unit is $\mathrm{m}$; $h$ is the suspension height of the linear luminaire in the calculated horizontal plane, the unit is $\mathrm{m}$; $d$ is the distance from the point $\mathrm{P}$ to the projection of the light source on the horizontal plane, the unit is $\mathrm{m} ; F_{x}$ is the azimuth coefficient, it can be found by looking up the table.

\section{Establishment of personnel position and mobility model}

Generally, the running of buildings is closely related to the number of personnel and the position of personnel, which are the important and fundamental information sources. As illustrated in the research ${ }^{[7-13]}$ that intelligent control based on personnel positions can save $10 \%$ to $30 \%$ energy consumption, especially in a large space building with great number or distribution of indoor personnel. Combined with the personnel position and movement model, in this paper, we introduced the intelligent lighting control simulation system regardding the distribution of personnel in the office. In addition, the personnel position and movement model provides data source for the building intelligent energy-saving and control system.

\subsection{Description of the personnel movement process}

The movement of personnel in office buildings has certain regularity. In fact, workers go office in a period of time, and stay in the office at working hours. These regular events repeatedly every day determine the regularity of the work and movement of the office staff. There are two main reasons for the change of the personnel's position: First, the movement of personnel from the external space to the office area or the opposite movement caused by the commute event; second, there is no incident, the movement of personnel due to workneeds and random-walking. Fig 4 shows the flow chart of the movement of staff.

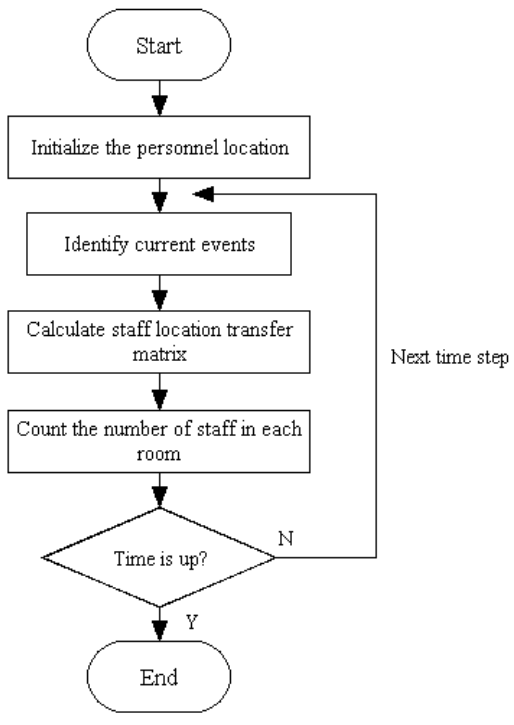

Fig.4.The flow chart of the movement of staff 
The staff movement simulation follows three general steps of simulation calculations: set initial parameterscalculate simulation-output calculation results. The position of each person is updated by the predicted data obtained by the position of personnel and the transfer matrix. After that, we can obtain all the personal information and indoor information from the statistical results.

\subsection{Algorithm implementation of the personnel movement process}

The input parameters of the personnel movement simulation include: time period, time step required for simulation, movement events, attributes and personnel in each room; the output is the position and the number of personnel in the room within each time step. The specific steps of the calculation of the personnel movement simulation are as follows:

1)Initialize the position of the personnel: set position status of all personnel at time 0 . For office building, set outdoor and each office from 0 to $n$.

2)Determine the active event-set at the current moment: start and end time of the event confirms the active state of event. Then, update the current active event collection;

3)Calculate the personnel transfer matrix at the current time: setting and updating related elements of the personnel transfer matrix according to the characteristic parameters of the active event;

4)Calculate personnel position: from the position of the previous moment and the updated transfer matrix, update the position of each personnel at the current time by probability;

5)Count the number of personnel in each room based on position;

6)Return to step 2), enter the calculation of the next moment.

Through repeating the above steps, we can obtain the position sequence of each personnel and the personnel status of each room, as well as the moment when each event occurs. These results can be further substituted into the simulation model of the use of lighting equipment. The model is not limited by the number of rooms and the layout of the room, and can be used for building of different scales; Therefore, we can obtain the information of each room and the movement path of each person.

\section{Simulation design of lighting control system}

The natural illumination model and the personnel movement model obtained in the above are used as input parameters of the intelligent lighting control system, the office lighting system is intelligently controlled in each time step according to the lighting conditions of the current time and the position of the personnel.

The above two models are used as input parameters of the lighting control system in the form of a matrix of time-by-time. The simulation system monitors the minimum illumination of the current office work surface and the personnel of the office area at each moment. With time goes by, when there is office staff in the office and the minimum illumination of the work surface is lower than the demand standard value, the lighting control system will turn on the light in the lowillumination area to meet the staff's need for office lighting; if the illumination meets the current environmental requirements, the system will turn off the light of the corresponding area to save power consumption; when the time slice runs out, that is, after the office worker leaves, the system will turn off all lights and exit.Fig 5 shows the flow chart of lighting control.

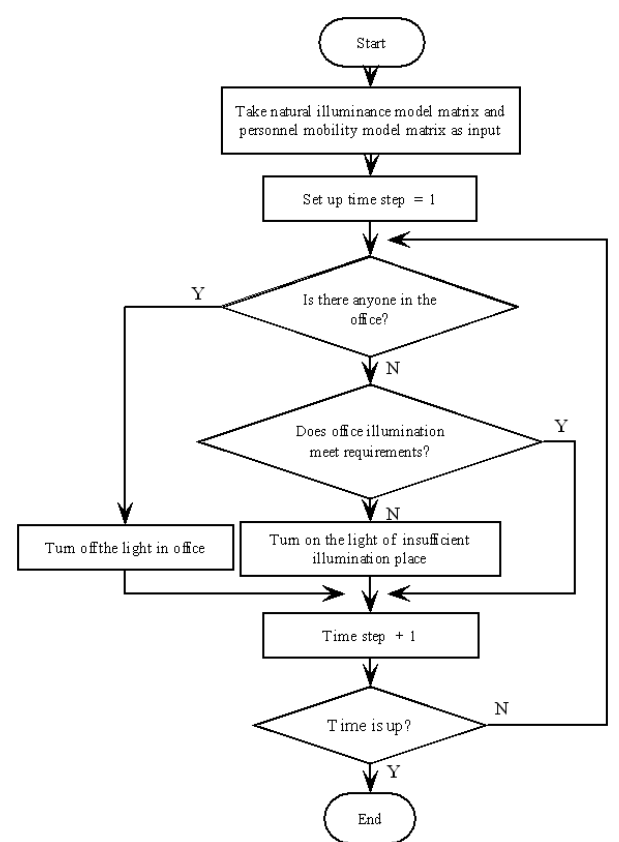

Fig. 5.The flow chart of lighting control

We take a real building office as a simulation example, the room is facing south, its length $M$ is $6 \mathrm{~m}$, width $N$ is $8.5 \mathrm{~m}$, height $H$ is $3.75 \mathrm{~m}$; the size of the window is $2.5 \mathrm{~m}^{*} 2 \mathrm{~m}$, the height of the lower edge of the window is $1.2 \mathrm{~m}$ from the ground, the distance $l$ from the corner of the room is $3 \mathrm{~m}$; the height of the office work surface is $0.75 \mathrm{~m}$. Then the illumination value of each point on the office work surface can be calculated according to the mathematical model in section 1 of this paper, in practical applications. In order to not only ensure accurate description of the illumination, but also to make the amount of data as small as possible for calculation, we set up a monitoring point every $10 \mathrm{~cm}$, then the lighting of such a $6 \mathrm{~m} * 8.5 \mathrm{~m}$ room is transformed into a $60 * 85$ illumination field matrix. Fig 6 shows the isolux graph of natural light when the luminous intensity of the light source is $1800 \mathrm{~cd}$. 


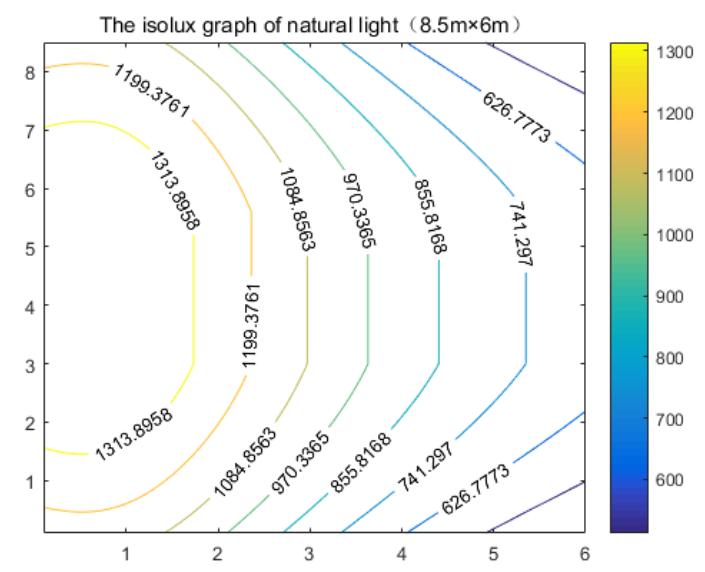

Fig. 6. The isolux graph of natural light

In this simulation system, we simulate the movement of personnel with a real office area. Fig 7 shows the plan view of the office area, there are 6 offices in this area with 10 personnel, of which office staff No.1, No.2 and 3 work in Room 401, staff No.4 work in Room 402, staff No.5 and 6 work in Room 403, and staff No.7, No.8 and 9 work in Room 404, staff No.10 works in Room 407, and Room 407 is used as a conference room, usually no one is working. There are a total of 48 time steps in this simulation, the movement of the personnel is simulated and the number of personnel in each room is counted every 15 minutes. The first two time steps represent the time period for going to work, and the last two represent the time period for getting off work. Fig 8 shows the changing situation of the number of staff in 402 during these 48 time steps.

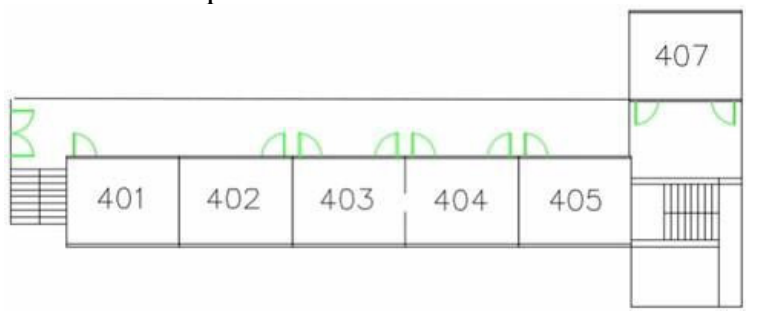

Fig. 7.The plane figure of office

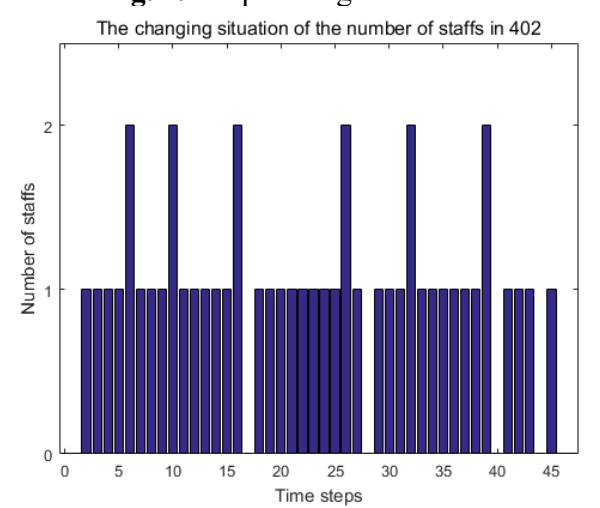

Fig. 8. The changing situation of the number of staff in 402

Fig 8 shows that, for Room 402, because staff enter the office from the outside during the period time of going to work(1-2 time steps in the figure), as well as the random walk during the normal working times(3-45 time steps), there may have more than one person or less than one person in the Room 402; during the off-work period(46-47 time steps), the office staff left the office and quited the random walk, there is no one in the Room 402.

According to the Architectural Lighting Design Standard GB50034-2013, the standard illumination value of the work surface of the general reading room and office $0.75 \mathrm{~m}$ from the ground is $300 \mathrm{Lx}$, therefore this simulation also uses $3001 \mathrm{x}$ as the standard to intelligently control the lighting system. We simulated the control of the lighting system during the day according to the input parameter processing method in Fig 5. Set the time step in the simulation to 15 minutes, so that it is divided into 48 time steps from 6 am to $6 \mathrm{pm}$. Fig 9 shows in 48 time steps, the switch state changes in three modes: constantlight mode, sensor-based control mode, the lighting control mode based on natural illumination and personnel movement model used in this paper. Constant lighting keeps the two-way lighting open; the light sensor-based control method will turn off the lighting to save energy when the illumination meets the requirements; and the control method used in this article will turn off all lighting when it detects that there is no one in the office to minimize the power consumption.

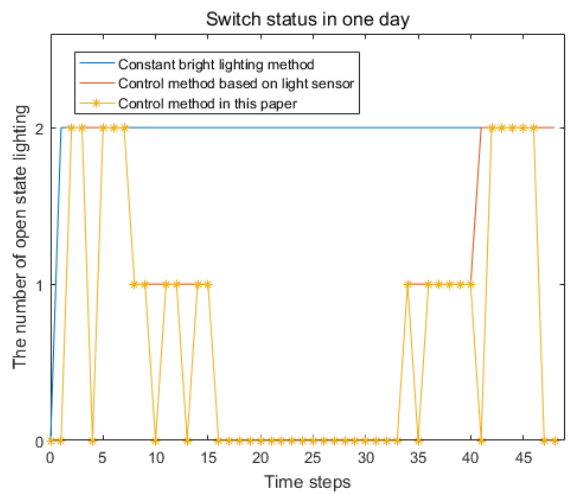

Fig. 9.The changing of the lighting switch of the day

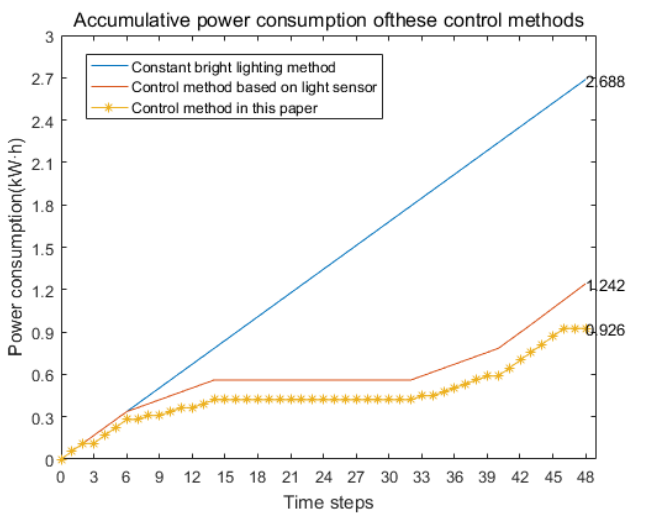

Fig.10.Accumulative power consumption of control methods

Fig 10 shows the cumulative power consumption of these three methods over these 48 time steps. It can be seen that the constant lighting mode turns on the lighting when the office staff arrives at the office, and turns off when the staff leaves after off work, the lighting energy consumption is on a straight line, eventually consuming $2.69 \mathrm{~kW} \cdot \mathrm{h}$. The ordinary light sensor-based control mode will turn off the lighting if the office illumination meets the requirements, from the above figure we can see that the power consumption is greatly reduced, and the final power consumption is $1.24 \mathrm{~kW} \cdot \mathrm{h}$. And the control method designed in this paper can regulate the real-time 
lighting according to the personnel's position, and it can save $25 \%$ energy saving compared with the light sensorbased control mode.

\section{Conclusions and prospects}

This paper designed an intelligent lighting control system based on the building non-central platform. Through simulating the perennal movement of indoors and the natural illuminantion of the office, the proposed intelligent lighting control system in this paper provides varied precise control of the light, which effectively use the natural light and reduce the waste of electric energy and prolong the service life of the lighting lamps. It can not only effectively use the natural light, but also reduce the waste of electric energy and prolong the service life of the lighting lamps. Furthermore a universal simulation model can be used for office lighting energy estimation in various layouts, which plays a guiding role in the actual lighting system design. Verified by the simulative experiments in this paper, the intelligent lighting control system is efficient and save more energy than the traditional lighting control system.

\section{Acknowledgments}

This work is supported by National Key Research and Development Project of China No. 2017YFC0704100 (entitled New Generation Intelligent Building Platform Techniques)

\section{References}

1. LeiPan, Dongqing Feng. The Research on the Control Strategy of Indoor Intelligent Lighting Combined with Natural Lighting. Control Theory and Control Engineering School of Electrical Engineering. May 2014.

2. Qi Shen. Research on the architecture of intelligent building non-central platform[D]. 2015.

3. Weiding Long. Building energy efficiency and building energy efficiency management[M]. China Construction Industry Press. 2005.

4. Fuyan Guo, Minde Huang. Building power supply and distribution and lighting[J]. China Construction Industry Press. 2014.

5. Aiying Wang. New progress of natural lighting technology. Journal of Architecture. 2003, 64-66

6. Rea MS, Bullough JD. A new framework for lighting practice. Lighting Research and technology[J]. 2001. Vol.19(2): 90-105

7. Kim Y S , Srebric J . Impact of occupancy rates on the building electricity consumption in commercial buildings[J]. Energy and Buildings, 2017, 138:591600 .

8. Shen W, Newsham G , Gunay B . Leveraging existing occupancy-related data for optimal control of commercial office buildings: A review[J]. Advanced Engineering Informatics, 2017.
9. Agarwal Y, Balaji B , Gupta R, et al. Occupancydriven energy management for smart building automation[C]. Acm Workshop on Embedded Sensing Systems for Energy-efficiency in Building. ACM, 2010.

10. K.A. Rahman1, A. Hariri, A.M. Leman, M.Z. Yusof. Energy consumption in residential building: The effect of appliances and human behavior. AIP conference proceeding. 2017.

11. FrédéricHaldi, DarrenRobinson. The impact of occupants" behaviour on building energy demand[J]. Journal of Building Performance Simulation, 2011, 4(4): 16.

12. Oldewurtel F, Sturzenegger D, Morari M. Importance of occupancy information for building climate control[J]. Applied Energy, 2013, 101(1):521-532.

13. Goyal S , Ingley H A, Barooah P . Zone-level control algorithms based on occupancy information for energy efficient buildings[C]. American Control Conference. IEEE, 2012. 